\title{
Research and Implementation of the Key Technology of UAV Aerial Image
}

\section{Transmission}

\author{
Fan Yijun ${ }^{1,2}$ \\ ${ }^{1}$ No.1 Institute of Geological \& Mineral \\ Resources Survey of Henan, \\ Luoyang, 471023, Henan \\ ${ }^{2}$ Provincial Key Laboratory of Gold and Silver \\ Metallogenic Series and Deep Prediction, \\ Luoyang, 471023,Henan, China . \\ e-mail: fanyijun1681@163.com
}

\author{
Lai Yufeng \\ School of Computer Science and Engineering \\ Xi'an Technological University \\ Xi'an 710021, Shaanxi, China \\ e-mail: 604612675@qq.com
}

\begin{abstract}
With the maturity of UAV technology, especially the cost of the four rotor unmanned aerial vehicle is decreasing, the unmanned aerial vehicle (UAV) will be a trend in the future. At the same time, because ZigBee has the characteristics of low cost, low speed and short transmission distance, we use ZigBee to achieve the transmission of image files between UAV and ground receiving system computers. This paper introduces the system design and software program module hardware node design, the first use of ZigBee wireless network as a data link, and then collected by the UAV to the ground of the computer image transmission, in the process of transmission by subcontracting, merging, stop and wait Agreement to ensure the correctness of data transmission right. Through experimental test, the correct transmission of image files between UAV and ground end computer can be realized when the image data is less than 1024 Kbytes.
\end{abstract}

\section{Keywords-ZigBee; UAV; Mapping; Image; Transmission}

\section{INTRODUCTION}

At present, in the commercial mobile communication network coverage, the image can be transmitted through the $4 \mathrm{G}$ network [1]. However, in some specific cases, the commercial mobile communication network can not be used when there is a problem with the base station transmitting the signal or when it is far from the coverage area of the transmitting base station. The ZigBee technology can be a very good solution to this drawback, just a gateway node can self-organize a network, other nodes can join directly or through multi-hop approach to join the network.

This paper proposes a ZigBee-based image file transfer system. Although ZigBee has the disadvantages of small bandwidth and low transmission rate, it can achieve very good wireless data transmission in applications where the data size is general and real-time performance is not high. Based on the image transmission of UAV, this paper studies the data transmission process of image files.

\section{SyStem DESIGN}

The system is mainly composed of two parts, a UAV and a ground computer. The ground computer mainly realizes the display and image processing of the human-computer interaction interface, and the UAV mainly realizes the image acquisition and transmission. The routing node and the gateway node part of the system use the ZigBee CC2530 kit [2]. The CC2530 not only supports the practical application of the $2.4 \mathrm{GHz}$ IEEE802.15.4/ZigBee standard, but also enables the establishment of powerful networks. The structure of this system is shown in Figure 1

\section{A. Ground Computer}

The ground computer completes the processing of the received data and displays the human-computer interaction interface. It is based on visual $\mathrm{C}++6.0$ and MSComm.

The MSComm control receives data through the serial port and provides a serial communication channel for the application. It is a simplified Windows-based serial communication programming Active control provided by 
Microsoft Corporation. Two methods are provided to deal with communication problems. One is the event-driven method and the other is the query method. This paper uses the event-driven method. [3]Event-driven is a very effective way to handle serial port interactions. In many cases, events need to be notified when they occur.

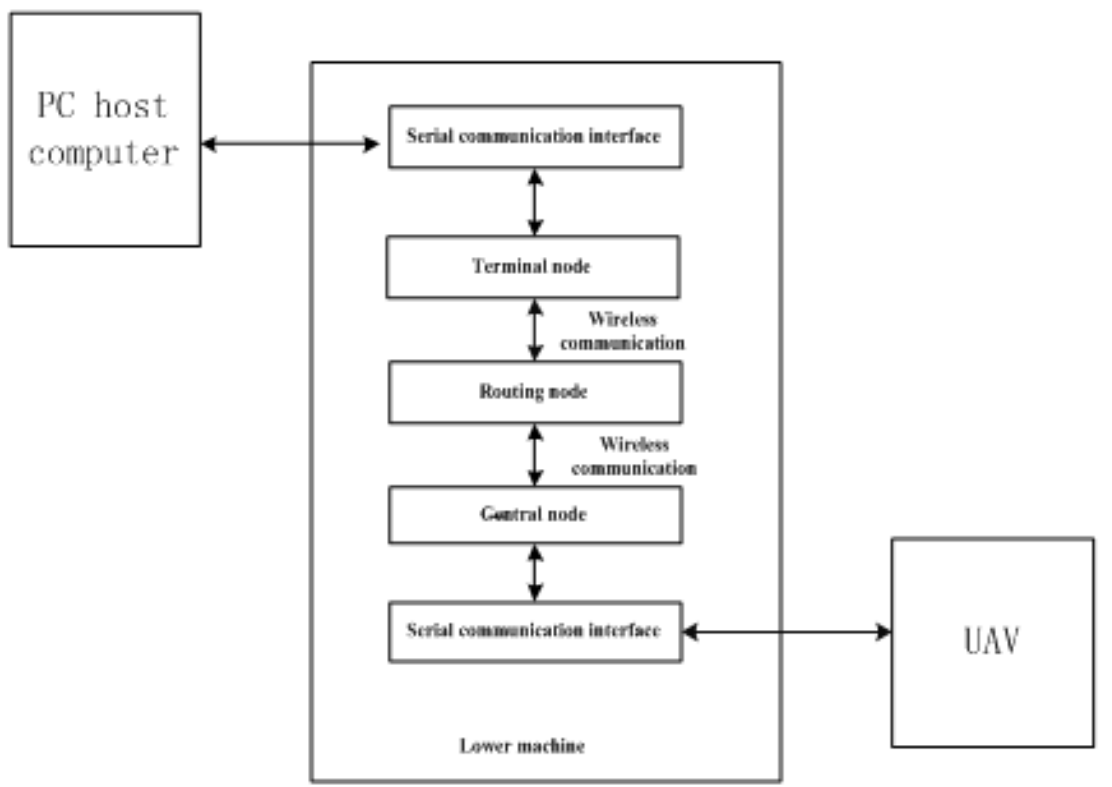

Figure 1. Structure of the system

\section{B. UAV System}

In this paper, UAV image transmission adopts the CC2530 kit of DTK Company, which uses three kinds of nodes: terminal node, routing node and central node (set the node of ZigBee module through DTK ZigBee Configuration and set it to the required node type. )[4]. The terminal node is connected to the UAV CPU through the RS232 serial port. After the UAV acquires the video data, it is transmitted to the terminal section through the serial port RS232. The data sent by the terminal node is received by the routing node. The central node is connected to the receiving end computer through the RS232 serial cable. The data sent by the routing node is received by the central node and transmitted to the ground computer through the RS232 serial port line. The UAV processes the image data, and then completes operations such as decoding and merging of the data. The receiver determines the next operation by using the human-computer interaction interface to determine the reception status of the image file [5].

\section{SOFTWARE IMPLEMENTATION}

The data transmission system of this paper is composed of three modules: data processing module, ZigBee data transmission module, data transmission and data reception. as shown in Figure 2.

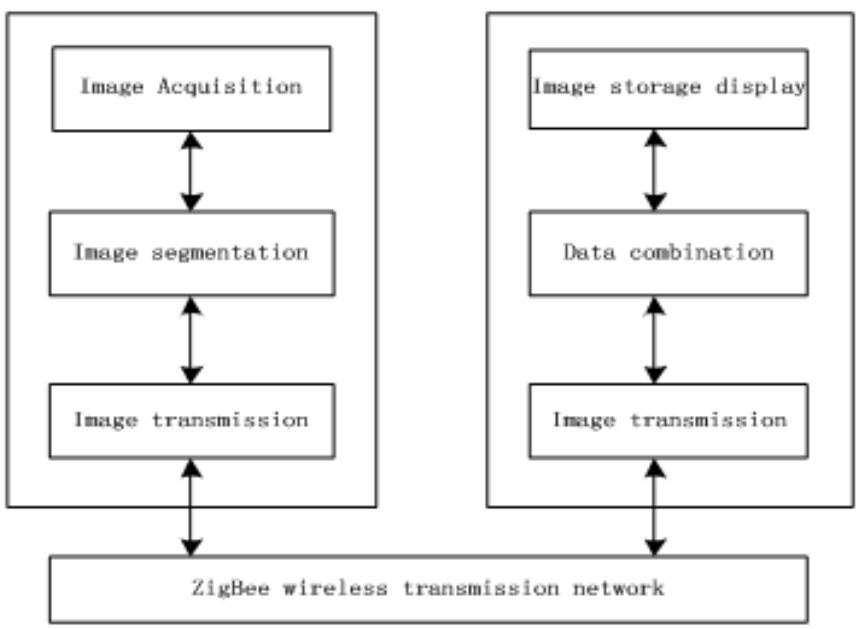

Figure 2. Module of Image transmission process 


\section{A. Data Receiver Module Programming}

The image receiving part is programmed by Visual C++ installed on the ground computer to process the received data to ensure the integrity and correctness of the data in the ZigBee wireless network [6].Obviously, when the set packet is larger, the data transmission time is shorter, and on the contrary, the transmission time is longer. The size of the package can be set according to actual needs. When the timeliness requirements are relatively high, the package can be divided into larger points. When the accuracy is required to be higher, the package can be smaller.

\section{B. ZigBee Data Transmission Module Programming}

ZigBee data transmission module realizes the function of correct data transmission. When the sending end computer sub-packets the data and names each package, it will connect the sub-packages in turn, and they are connected through the ZigBee module connected to the RS232 serial port. Data is transmitted in a ZigBee wireless network. Data is transmitted to a routing node through a terminal node and finally to a central node. The central node will receive each packet in sequence and transmit the data to the receiving end computer through the RS232 serial cable. The receiving end computer performs a series of operations on the uploaded data, including decoding, merging, storage, etc., and finally completes the data transmission operation.

\section{Data Selection and Saving Module Programming}

When the UAV sends data, first, the file is compressed and sub-packaged at the sender, and then transmitted in turn according to the compression algorithm. The receiving end computer receives the uploaded sub-packets in sequence and compares the received sub-packing sequence number with the local receiving state variable. In the first case, the two are equal, indicating that this is a new data packet. In this case, the receiving end computer receives the data packet and sends the confirmed message. The second case is that the two are not equal, the receiving end computer directly discards the sub-packet, and then sends a retransmission message to the sending end. The correctly received subcontracts are subjected to the following operations, including unpacking, saving, and merging, and are named before the "-", and then the entire file is saved in jpg format. The receiving progress of the file and the preservation of the file can be seen on the human-computer interaction interface of the receiving end computer. If you want to find a file that has been received, you can open it by finding the location where you want to save the file on the host computer. You can view and edit it.

\section{DATA FRAME FORMAT}

The ZigBee network uses the center coordinator to create the network. When a node joins, it assigns addresses to the joining nodes so that they can form a star network, a tree network, and a mesh network. The required networks can be organized as needed so that the nodes joining the network can communicate with each other.

The ZigBee network supports the mutual transmission of data between any node in the network. Data transmission modes between nodes include transparent transmission and point-to-point transmission.

\section{Key TeChNOLOGIES}

This paper adopts key technologies such as image subcontracting and merging between a UAV and a ground computer. It has the advantages of low cost and simple operation, and is characterized by safe, stable and cost-effective file transfer.

\section{A. Document Subcontracting Process}

When the UAV sends an image, it first compresses the image file and then calculates the number of bytes of the compressed file. If the data volume does not exceed the sub-packet limit, the data is sent directly. If the file data volume is greater than the sub-packet limit, the data should be sub-packaged. The process of file subcontracting is as follows. First, the name of the file is obtained, and the file name is placed in the first package for transmission. The name of the file should not exceed the upper limit of the packet, which is 73 bytes, telling the receiver that the sender will send the file with this name. Then, according to the actual situation, the file is sub-packaged, and the sub-packet

file name is changed to the form of <"source file name"+"-"+"divided serial number i">. This cycle continues 
until the subcontracting operation is completed and the sub-packages are saved in the folder specified by the computer. After the subcontracting is completed, the divided data packets are transmitted through the ZigBee wireless network. As shown in Figure 3.

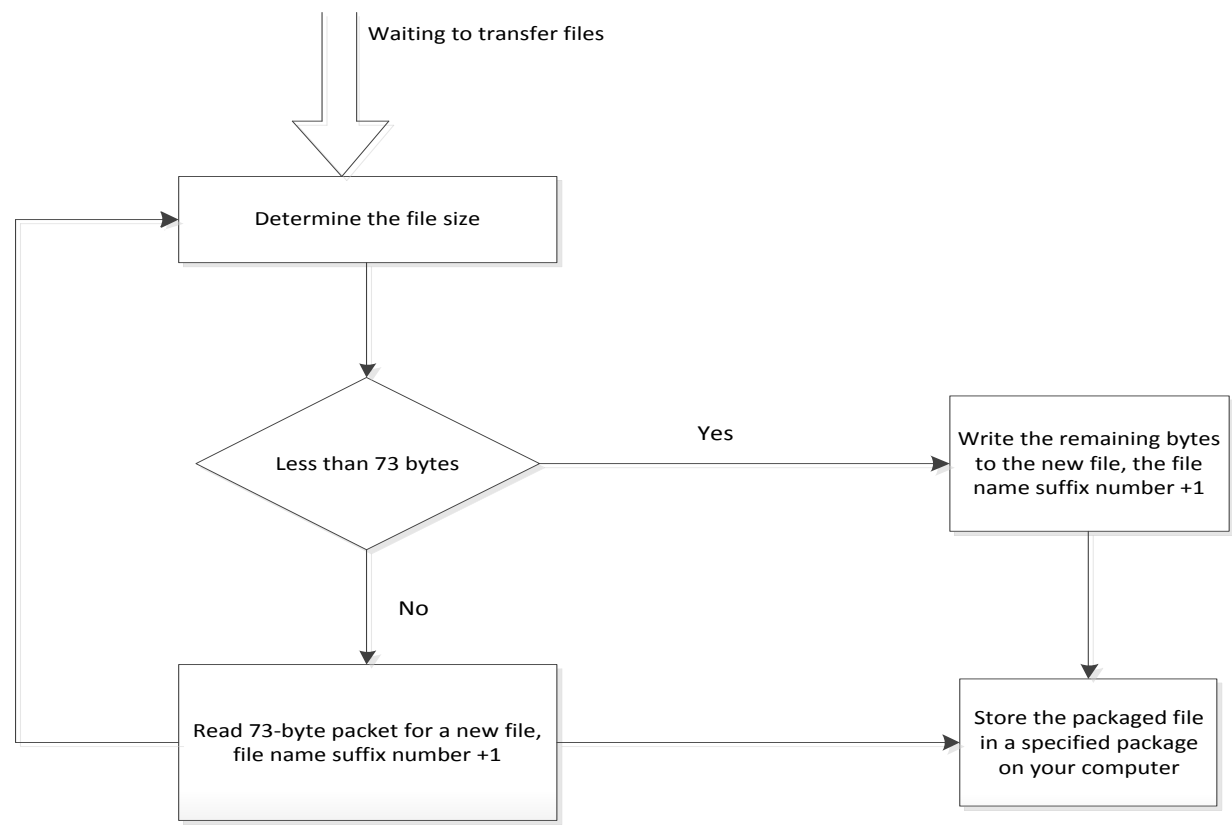

Figure 3. Process of image file subcontracting

\section{B. Stopping the Waiting Protocol}

After the file subcontracting process is completed, the subcontracted files are sequentially transmitted in order. In order to ensure the correctness of data transmission, the receiving end computer sends a confirmation message after receiving the subcontracting. According to the received confirmation message, the UAV determines whether the subcontracting is successful and whether it needs to re-send the subcontract. If the transmission succeeds, it sends the next packet and deletes the packet that was sent successfully. If it fails, it resends the packet until it is sent successfully or reaches the set upper limit [7]. So how does the computer perform the above operations? It is to stop waiting for the agreement.

The stop waiting protocol means that after the small packet file is transmitted to the receiving end through the ZigBee wireless sensor network, it will send a new packet only after receiving the confirmation packet with the correct sequence number, otherwise it will resend the data packet just sent.

\section{File Consolidation Process}

The sub-packed image files are received by the receiver in sequence according to the transmission order after the stop wait protocol. The receiving end saves the received file in a folder specified by the computer and merges the small file into a large file. The large file is named with the "file name" of the small file name. After each successful merge, the received new file is packetized and deleted. Save the merged file in a specified location on your computer. Looping like this to complete the merging of files completes the reception of the entire file [8].

\section{SySTEM TESTING}

The system uses a UAV and a ground computer to achieve system functions. First use a RS232 serial cable to connect a ZigBee communication module to the receiving computer. If the tested UAV is far away from the computer, you need to set up a routing node to relay the sent file. If the UAV is close to the computer, there is no need to set up. A terminal node or a central node can complete the system test. 
Then power on the ZigBee module. After the system is powered on, configure a star network according to the settings to implement the wireless data transmission function. The resulting hexadecimal code is shown in Figure 4. After clicking the display button, the actual image after conversion appears. The number of pixels in the image is $339 * 272$. The image size is about $90 \mathrm{~KB}$. The image is clear and the effect is good, as shown in Figure 5. In the case that the resolution requirement is not high enough, it meets the general requirements for reading geological features.

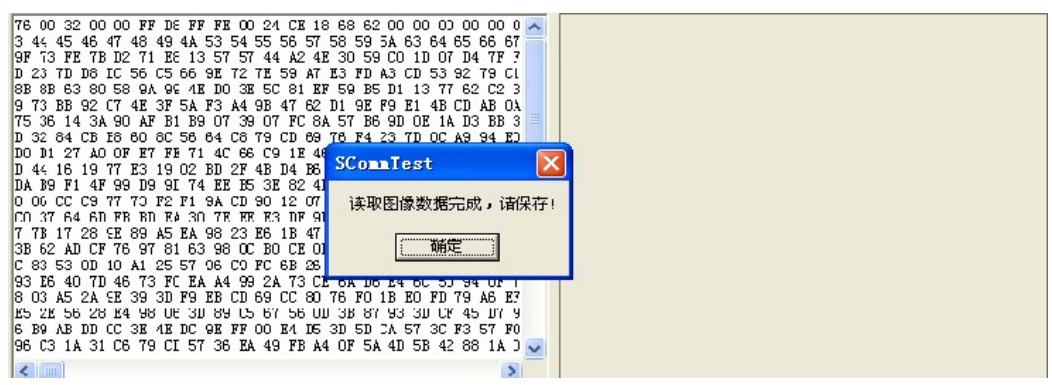

Figure 4. Image hexadecimal representation figure

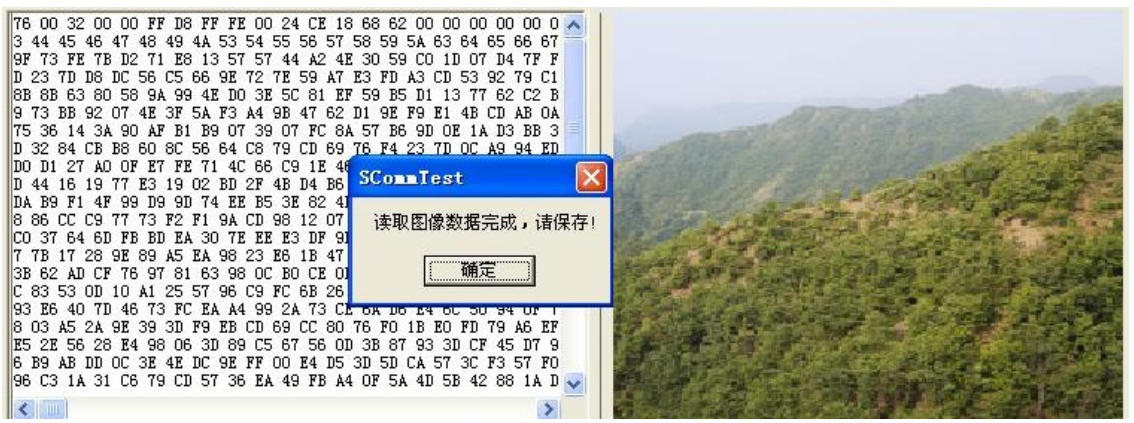

Figure 5. Image display figure

\section{CONCLUSION}

In this paper, based on the study of the characteristics of ZigBee wireless network, a wireless image transmission system of UAV based on ZigBee is proposed. Through the system design, the workflow of the whole system is introduced, and the hardware design, software design, key technologies of data transmission and system testing are given. The hardware design includes hardware selection and hardware implementation. Software design includes data processing, ZigBee network and human-computer interaction interface. Key technologies introduced subcontracting, stop-waiting protocols, and mergers. Finally, the function of this system was introduced, and the "image transmission" function of the system was tested. This system is based on the simple and flexible ZigBee wireless network. It has the characteristics of low hardware cost, simple software programming, and has a certain application prospect.

\section{REFERENCES}

[1] Lu Zhian. ZigBee network principle and application development [M]. Beijing: Beijing University of Aeronautics and Astronautics Press, 2008.

[2] Yang Fubao. Research design of data transmission system based on ZigBee[D]. Wuhan University of Technology, 2011.

[3] Yao Zhandong. A LAN-based File Transfer Technology. Computer Applications, 2004: 45-47.

[4] Cai Wenjing. Wireless data acquisition system based on ZigBee technology[D]. Hangzhou Dianzi University, 2011:1-5.

[5] Z. Q. Song, X. Z. Zhou, and L. Wang, "Research on wireless image transmission based on ZigBee," Computer Applications and Software vol. 29, no. 7, pp. 1-5, 2012.

[6] J. Lloret, I. Bosch, S. Sendra, and A. Serrano, "A wireless sensor network for vineyard monitoring that uses image processing," Sensors, vol. 11, no. 6, pp. 6165-6196, 2011.

[7] D. G. Lowe. Distinctive Image Features from Scale-Invariant Keypoints[J]. International Journal of Computer Vision.2014, 60(2): 91-110.

[8] C. Harris, M. J. Stephens. A Combined Corner and Edge Detector[C]. Prco of the 4th Alvey Vision Conf, 2015 